\title{
Blood Sugar, Your Pancreas, and Unicorns: The Development of Health Education Materials for Youth With Prediabetes
}

Lisa G. Yazel-Smith, Julie Pike, Dustin Lynch, Courtney Moore, Kathryn Haberlin, Jennifer Taylor, Tamara S. Hannon

\begin{abstract}
Background: The obesity epidemic has led to an increase in prediabetes in youth causing a serious public health concern. Education on diabetes risk and initiation of lifestyle change are the primary treatment modalities. There are few existing age-appropriate health education tools to address diabetes prevention for high-risk youth.
\end{abstract}

Aim: To develop an age-appropriate health education tool(s) to help youth better understand T2D risk factors and the reversibility of risk.

Methods: Health education tool development took place in five phases: exploration, design, analysis, refinement, and process evaluation.

Results: The project resulted in 1) booklet designed to increase knowledge of risk; 2) meme generator that mirrors the booklet graphics and allows youth to create their own meme based upon their pancreas' current mood; 3) environmental posters for clinic, and 4) brief selfassessment that acts as a conversation starter for the health educators.

Conclusion: Patients reported high likability and satisfaction with the health education tools, with the majority of patients giving the materials an " $\mathrm{A}$ " rating. The process evaluation indicated a high level of fidelity and related measures regarding how the health education tools were intended to be used and how they were actually used in the clinic setting.

This is the author's manuscript of the article published in final edited form as: Yazel-Smith, L. G., Pike, J., Lynch, D., Moore, C., Haberlin, K., Taylor, J., \& Hannon, T. S. (2018). Blood Sugar, Your Pancreas, and Unicorns: The Development of Health Education Materials for Youth With Prediabetes. Health Promotion Practice, https://doi.org/10.1177/1524839918778555 


\section{Consistency with Journal Mission:}

This article is consistent with the mission of Health Promotion Practice as it addresses the development and implementation of health education tools for use in a clinical setting. The health education tools described herein focus on the prevention of type 2 diabetes in a priority population of high-risk youth.

\section{INTRODUCTION}

The obesity epidemic has led to an increase in type 2 diabetes (T2D), as well as the precursor condition "prediabetes" (Weiss, 2007). It is estimated that approximately $30 \%$ of U.S. children aged 12-17 years have prediabetes (Bullard, 2013).The number of youth diagnosed with prediabetes continues to increase, and as a serious public health concern, youth with prediabetes have significantly increased risk for developing T2D (Cali, 2009). The significant impact of modest weight loss and physical activity on the prevention or delay of T2D among individuals with prediabetes has been demonstrated (Savoye, 2014).Thus, for families of youth at risk for prediabetes and T2D, education on diabetes risk and initiation of lifestyle change are the primary treatment modalities.

It is important to have effective health education communication channels to foster positive changes in youth (DiMatteo, 2004). However, there is a lack of age-appropriate tailored health promotion and education materials to address diabetes prevention for high-risk youth in the clinical setting. The Diabetes Prevention Program (DPP), a randomized controlled trial that created the gold standard for diabetes prevention, was tested only in adults and not youth (DPP Research Group, 2004). Existing materials for youth prevention often have a very narrow reach, are designed for very specific populations, addresses young children, and are not distributed in the healthcare setting (Centers for Disease Control and Prevention, 2015). Additionally, there is 
limited available materials aimed towards older youth, who have more autonomy over their health behaviors (Stinson, 2009).

Clinicians and health educators need tools to effectively educate youth and families on diabetes progression and communicate diabetes risk reduction, as the main goal of clinical health promotion is to enable patients to take control of their own health (Kok, 1997). Tailored health education materials have been shown to be an effective means of health education in making lifestyle changes in comparison to generic resources (Krueter, 2000; Skinner, 1999; Noar, 2007). It is important to ensure that patients receive consistent information using age-appropriate concepts. Successful education requires youth be willing to learn about a disease in order to prevent or delay onset (Stinson, 2009), but barriers such as comorbid health conditions, low socioeconomic status, overburdened familial roles, lack of access to health education, and the cost of medical care can factor into the ability to understand the disease and prevention strategies (Stinson et al, 2009). Pairing education with a multi-disciplinary team may help overcome these barriers and lead to better adherence to behavior changes (Lange, 2007).

Traditionally, patient health education focuses on disease management and health promotion focuses on primary prevention (Caraher, 1998). The Youth Diabetes Prevention Clinic (YDPC) at Indiana University Health merges these two disciplines to ensure a wellrounded approach to diabetes prevention for at-risk youth and their families. Patients receive both medical care from the clinic team and health education from health educators. Therefore, staff at YDPC, which consists of the clinic medical director, staff physician, registered dietitian, and certified health education specialist, collaborated with a professor at Herron School of Art and Design at Indiana University Purdue University Indianapolis, and two professional communication designers to work as a research team on this project. While considering the 
guidelines published by the American Diabetes Association and U.S. Centers for Disease Control and Prevention, the purpose of this project was to develop a medically correct and effective health education tool(s) for communicating the importance of T2D prevention while using tailored, youth appropriate language, visually exciting graphics, and relevant youth-friendly design. Subsequently, the research team conducted a process evaluation of the health education tools to ensure standard use. The researchers hypothesized that clinic staff engagement and a patient-engaged development process would result in a patient-friendly, factually informative, and innovative original health education tool(s) to address T2D risk factors and modifiable lifestyle changes.

\section{METHODS}

The YDPC is a specialty clinic treating youth with prediabetes and risk factors for the development of T2D. (Insert Table 1 here) YDPC provides a comprehensive medical exam; point-of-care laboratory testing including two-hour oral glucose tolerance test, fasting glucose level, hemoglobin A1C, cholesterol levels; a dietary consultation; and health education at the initial clinic appointment. Patients know all lab results prior to leaving their clinic visit. Those with prediabetes return to clinic every three to six months for follow-up labs, clinical exam, dietary consult, and health education; those who do not have prediabetes are referred back to their primary care provider with a plan for reducing risk for T2D.

After reviewing the existing health education materials, the research team determined a gap in patient education options that presented accurate information for the at-risk youth population, while engaging the population with age-appropriate language and graphics. The communication designers decided to work through the design of the new potential health education tool(s) in a streamlined way that allowed succinct steps to ensure data would be 
collected to produce reportable results. The health education tool development took place in five distinct phases: exploration, design, analysis, refinement, and process evaluation. This study was approved by the Indiana University Institutional Review Board.

\section{Exploration}

The first step in the process was to define the specific challenge to address. This began by gathering data through YDPC staff key informant interviews, a clinic walk through by the communication designers, and a review of existing T2D-related health education materials.

Staff key informant interviews. Using an inductive method of data collection, the communication designers conducted key informant interviews with the clinic medical director, clinic staff physician, dietitian, health education specialist/data manager in order to understand the challenges to be addressed by the potential health education tool(s). The communication designers needed to gain an understanding of what the staff key informants wanted patients to know with regard to T2D prevention. Additionally, the staff members are content experts and could educate the communication designers on the various aspects of prediabetes, T2D, prevention, and clinical efforts. (Insert Table 2 Here)

Clinic walk through. The communication designers spent time in YDPC in order to witness the patient clinic experience from the patient's perspective. The communication designers started with the patient check-in process and followed the path that each patient makes once called into the clinic area. Guided by a clinic staff member, the communication designers focused on the physical environment of the clinic space as well as details of the amount of time patients spend at each stop (e.g. patient check-in, vital sign check, laboratory waiting area, exam room, and checkout). The communication designers were interested in finding potential points of 
intervention during the clinic visit. The observations were open ended with no official documentation of the findings other than personal synthesis by the communication designers.

Review of existing patient education material. The communication designers reviewed available existing prediabetes and T2D prevention health education material, including websites and materials from organizations such as American Diabetes Association (ADA, 2017) and the National Institute of Diabetes and Digestive and Kidney Disease (2016). The communication designers examined the existing materials to determine the intended audience for the material, the reading level of the material, if graphics seemed relevant to youth compared to popular trends, and how the author disseminated the material to patients or the public.

\section{Design}

The communication designers synthesized findings from the exploration phase and continued to examine other sources of information to inform the design needs. For instance, the designers formed ideas based upon their review of current trends in social media that may appeal to youth, such as memes, social media platforms, infographics, and video games. The designers explored multiple ways to address prediabetes and risk factors for the development of T2D in youth while focusing on how to accommodate the need for individual patients in different stages of change with regard to prediabetes and $\mathrm{T} 2 \mathrm{D}$ prevention.

\section{Analysis and Refinement}

With four draft tools in hand, a clinic staff person approached a convenience sample of 15 clinic patients over a series of multiple clinic dates in order to field test the health education tools. These patients were selected because they were seen in YDPC during the timeframe that fit the design needs. For those who gave verbal consent, the staff person then asked a series of questions to gauge the youths' perception of the developing health education tools. (Insert Table 
3 here) The project team made final revisions to the health education tools in order to accommodate the patient responses.

\section{Process Evaluation}

The research team conducted a process evaluation of the health education tools with 30 new patients to ensure standardized use. The patients were a convenience sample who attended YDPC appointment in an eight-week period. The process evaluation focused on the standard operating procedures (SOP) for use of the health education tools. The researchers used an exploratory evaluation design to assess the implementation process of the health education tools. Information was collected using a patient checklist and an observation checklist. The patient checklist documented the steps each patient goes through from receiving the booklet and selfassessment, to reading the booklet and completing the self-assessment, to discussing these items with the health educator. A YDPC staff observer used the observation form to record each step as either complete or not complete based upon patient recall. The answers from both forms were then matched together to ensure the steps were being completed as required in the standard operating procedures. Any discrepancies were discussed and reconciled with the health educator.

During each patient appointment, the researcher used the forms to measure fidelity of the health educator's instructions, to evaluate the dose delivered by the educator, to evaluate the dose received by the patient, to record the length of time the health educator spent with each patient, to assess the patient's risk based upon the risk graphic in the booklet, and the knowledge of reversibility of prediabetes. The researchers monitored reach by tracking the number of patients approached. Researchers evaluated context by asking patients if they had received diabetes prevention education prior to the YDPC visit. If yes, they were asked to identify the nature and location of information (e.g. school, primary care doctor, community program). 


\section{RESULTS}

The research team developed the following challenge statement: How might providers engage high-risk youth who are seen in the diabetes prevention clinic in order to 1) introduce them to diabetes prevention as being on a continuum and 2) encourage them to take ownership of their health behaviors without 3 ) causing them to be fearful, to shut down, to feel embarrassed, or to feel overwhelmed? Based upon the findings of the staff key informant interviews, clinic walk through, and review of existing patient education material, the research team developed four distinct health education tools, namely a booklet, a meme generator, clinic environmental posters, and a brief self-assessment for patients.

\section{Exploration}

Staff key informant interviews. Four staff key informant interviews were conducted during the initial exploration phase. The main outcome of the key informant interviews was that patients need to know the facts about how the pancreas affects the development of T2D while understanding that T2D prevention and development is on a continuum. (Insert Table 4 Here)

Clinic walk through. During the clinic walk through, the designers found that some patients were in clinic for up to four hours. During this time, patients are checked-in, roomed, have labs drawn, have a physical exam by the physician, and have dietary and physical activity consultations with the dietitian and/or health educator. However, down time in the exam room allows opportunity for patients to get bored, frustrated, worry about results. Therefore, patients may not be fully receptive to receiving lab results or new information about lab results at the end of a long appointment. During the clinic walk through, the communication designers determined time spent in the clinic room could be an opportune time to intervene with patients. Therefore, exam room environmental posters were designed to act as an intervention vehicle. The patients 
have time to read the environmental posters and interact with them alone as well as with the clinic staff during the appointment. Additionally, the environmental posters can get the patients involved in the conversation because he/she can move around the room to discuss each risk level with clinic staff.

Review of existing patient education material. The communication designers found that many already-existing pieces of information are factually accurate. However, they gloss over the importance for high-risk individuals to take control of their health behaviors and promote little incentive to patients for changing their lifestyles. In addition, some resources did not include reference to contributing lifestyle factors, and few pieces were geared toward youth. Additionally, the existing materials did not address the idea that T2D is on a continuum.

\section{Design}

In order to meet the challenge statement, the research team employed the Transtheoretical Model (TTM) and the stages of change to frame the health education content and communication methods used to develop he health education tools. The TTM was chosen because it helps the priority population progress from their current stage toward adopting a health behavior change. The health education tools were designed to show the continuum of the progression of T2D as well as the reversibility of the progression of T2D. Employing the TTM in the design of the health education tools allows patients to understand how making changes in their behaviors can change the trajectory of T2D.

Simultaneously, the YDPC clinic staff developed the clinical educational content regarding diabetes prevention, lifestyle changes, and goal setting strategies. The entire research team then revised the content graphics to fit into the overall desired tone of the health education 
tools as well as the feedback from the 15 YDPC clinic patients that assessed the health education tools.

Booklet. The booklet titled Why are you testing me for diabetes: A booklet about blood sugar, your pancreas, and unicorns was developed as the main health education tool for use in clinic. The research team felt a booklet would be the best media option because the patients can take it home for future reference, and a booklet allows for the clinic staff and patient to write notes and comments. The content of the design uses non-technical language to introduce the physiologic components of diabetes, the difference between type 1 and type 2 diabetes, and risk factors for the development of T2D. The health education booklet tool then moves into content to help patients understand their individual risk for the development of T2D, how the patient can reduce risk, and goal setting based upon their stage of change. The health education booklet introduces diabetes prevention using personification of a pancreas using art style like popular cartoons, and by focusing on the pancreas as the physiological mechanism of T2D. The final version is a 16-page health education booklet tool that is introduced to new patients at the beginning of each new patient clinic visit.

Meme generator: A web-based meme generator was developed as a supplemental activity for patients to use to document their progress with making behavior change. Patients can choose a pancreas "mood" and add a caption to represent their own mood at that time. It is introduced to the patients for their use outside of clinic visits. (Insert Figure 1 Here)

Environmental posters: The environmental posters are a way to reinforce the messaging that patients received. There are four visual posters, and they are designed with the pancreas mood risk levels. Each poster is approximately 12 inches in diameter and can affix to the wall with removable sticky tabs. The communication designers determined this to be a good way to 
intervene as the patients spend a great deal of time in the exam room waiting for lab results. The posters offer an additional way to drive home the effect of behaviors on risk factors. They are used as a health education tool with the patients and family members to help visually explain risk levels and lab testing results.

Self-assessment: The self-assessment is comprised of nine questions that lead the patient to a score by personality in the booklet; the three characters represent how the patient treats his or her pancreas: 1) unicorn of awesomeness (high score), 2) that one guy (mid score), and 3) infinity math homework (low score). The self-assessment, which assesses patients' nutrition and activity behaviors, acts as a conversation starter between the health educator and the patient. Additionally, the self-assessment allows the patient to have ownership in the conversation regarding his/her risk.

\section{Analysis and Refinement}

Analysis and refinement took place after the design steps were completed. To refine the health education tools, 15 patients were engaged in a brief conversation with a clinic staff person regarding the design of the health education tools. Patients overwhelmingly liked the use of the animated pancreas used throughout the booklet, meme generator, and environmental posters. Additionally, the patients liked the self-assessment result categories that placed them into a continuum based upon their self-assessment answers. Patients requested two revisions. Patients wanted more explanation of the lab tests performed in clinic. The research team added brief explanations for the lab tests and results to the booklet. Second, patients wanted more information about what to eat and what not to eat in the booklet. The research team decided to not include more information on this topic because it would create a longer booklet and each patient gets a dietary consultation and goal setting with the dietitian. (Insert Table 5 here) 


\section{Process Evaluation}

Process evaluation observations were completed with 30 individual new patients. The process evaluation specifically focused on the booklet and self-assessment. The research team expected $95 \%$ fidelity, dose delivered, and reach due to the small sample size, the potential of an inappropriate referral to the clinic (a patient at normal risk T2D), or a family that does not speak English, as the health communication tools were designed in English. (Insert Table 6 here)

\section{DISCUSSION}

The research team developed age-appropriate health education tools to be used in a clinic setting with youth at risk for the development of T2D. Findings show there are no publically available appropriate health education tools or resources for this specific population. The research team found that engaging youth and clinic staff to develop health education tools is an appropriate way to address this need. Results indicate clinic staff wanted patients to know that the T2D trajectory is reversible. Youth reported they wanted health education tools that reflect ageappropriate design. Additionally, the clinic staff completed a process evaluation to ensure the standardized use of the health education tools.

The main outcome of this project was the development of the health education tools. The current literature suggests that tailored health education tools are more effective then generic sources (Krueter, 2000; Skinner, 1999; Noar, 2007). The health education tools were designed to be tailored to the risk of the individual clinic patient. More than $90 \%$ of youth ages 12 to 17 engage in some form of social media (Lenhart, 2015); therefore, the meme generator was developed as a way to connect with youth via social media outlets. Additionally, the final health education tools were designed to address the lack of health education pieces available to this 
population, as this presents a barrier to learning about successful behavior modification (Stinson, 2009).

The process evaluation of the use of the health education tools in clinic was successful as the health educator engaged the patients with the health education tools as directed in the SOP. Additionally, patients also interacted with the health education tools in a way that the research design team had hoped in the development phase.

With the increasing number of youth with prediabetes and T2D, it is important for health educators to engage patients and families in lifestyle behavior change discussions. The results of this project meet this need. Additionally, using tailored health education tools with this specific demographic population is beneficial to discussing behavior change. This is an example of how pairing population-specific health education tools with a clinical team is successful in educating at-risk youth (Lang, 2007).

The health education tools may have limited generalizability as they were developed for a specific specialty diabetes prevention clinic setting. However, the artifacts may be generalizable to other similar settings that serve similar patient populations. Additionally, the research team members appreciate that not all clinical staff teams have access to collaborative design professionals who specialize in health education messaging. Utilizing communication design experts to improve the development and dissemination of clinical research findings and to translate research effectively into clinical practice should be considered when developing new material. The findings of our process evaluation are limited in terms of generalizability since the health education tools were tested in one specific diabetes prevention clinic and the youth participants were a convenience sample who were available to review the information on a specific day. Finally, the process evaluation was limited by self-reported patient responses. 
However, the diverse youth in clinic may extend the generalizability of this study. The next step for this project is to test the use of the health education tools in community-based settings. 


\section{REFERENCES}

American Diabetes Association (2017). Diagnosing diabetes and learning about prediabetes. Retrieved from http://www.diabetes.org/are-you-atrisk/prediabetes/?referrer=https://www.google.com/

Bullard, K.M., Saydah, S., Imperatore, G., Cowie, C., Gregg, E., \& Geiss, L. (2013). Secular changes in U.S. prediabetes prevalence defined by hemoglobin A1c and fasting plasma glucose: National Health and Nutrition Examination Surveys, 1999-2010. Diabetes Care 36(8), 2286-2293.

Cali, A.M., Man, C.D., Cobelli, C., Dziura, J., Shaw, M., Allen, K., Chen, S., \& Caprio, S. (2009). Primary defects in beta-cell function further exacerbated by worsening of insulin resistance mark the development of impaired glucose tolerance in obese adolescents. Diabetes Care 32(3), 456-461.

Caraher, M. (1998). Patient education and health promotion: Clinical health promotion-the conceptual link. Patient Education and Counseling, 33(1), 49-58.

Centers for Disease Control and Prevention (2015). Eagle books toolkit. Retrieved from https://www.cdc.gov/diabetes/ndwp/eagle-books-toolkit/index.html

Diabetes Prevention Program Research Group. (2008). Achieving weight and activity goals among diabetes prevention program lifestyle participants. Obesity Research, 12(9), 14261434. 
Kok, G, van den Borne, B., \& Mullen, P.D. (1997). Effectiveness of health education and health promotion: Meta-analyses of effect studies and determinants of effectiveness. Patient Education and Counseling 30(1), 19-27.

Kreuter, M. W., Oswald, D. L., Bull, F. C., \& Clark, E. M. (2000). Are tailored health education materials always more effective than non-tailored materials? Health Education Research, $15(3), 305-315$.

Lange, K., Sassmann, H., Von Schütz, W., Kordonouri, O. \& Danne, T. (2007).

Prerequisites for age-appropriate education in type 1 diabetes: a model programme for paediatric diabetes education in Germany. Pediatric Diabetes, 8, 63-71.

doi:10.1111/j.1399-5448.2007.00277.x

Lenhart A. (2015). Teens, social media \& technology overview 2015. Retrieved from http://www.pewinternet.org/2015/04/09/teens-social-media-technology-2015/

National Institute of Diabetes and Digestive and Kidney Diseases (2016). Retrieved from: https://www.niddk.nih.gov/health-information/diabetes/overview/preventing-type-2diabetes

Noar, S. M., Benac, C. N., \& Harris, M. S. (2007). Does tailoring matter? Meta-analytic review of tailored print health behavior change interventions. Psychological Bulletin, 133(4), 673.

Savoye M., Caprio S., Dzuira J., Camp A., Germain G., Summers, C., Li, F., Shaw, M., Nowicka, P., Kursawe, R., Depourcq, F., Kim, G., \& Taborlane, W.V. (2014). Reversal of early abnormalities in glucose metabolism in obese youth: Results of an intensive lifestyle randomized controlled trial. Diabetes Care 37(2), 317-324. 
Skinner, C. S., Campbell, M. K., Rimer, B. K., Curry, S., \& Prochaska, J. O. (1999). How effective is tailored print communication? Annals of Behavioral Medicine 21(4), 290-298.

Stinson, J., Wilson, R., Gill, N., Yamada, J., \& Holt, J. (2009). A systematic review of internetbased self-management interventions for youth with health conditions, Journal of Pediatric Psychology, 34(5), 495-510.

Weiss, R. (2007). Impaired glucose tolerance and risk factors for T2DM in youth. Pediatric Diabetes 8(9), 70-75. 